\title{
REGISTRO, DOCUMENTACIÓN E INTERPRETACIÓN DEL ARTE RUPESTRE. UNA PROPUESTA BÁSICA PARA ESTUDIANTES: EL SEMINARIO TALLER DE LA SIARB
}

\author{
Registration, documentation and interpretation of rock art. \\ A basic proposal for students: the seminar workshop by SIARB
}

\author{
Matthias Strecker \\ Sociedad de Investigación del Arte Rupestre de Bolivia \\ (SIARB). La Paz, Bolivia. \\ Correo-e: strecker.siarb@gmail.com \\ Pilar Lima \\ Sociedad de Investigación del Arte Rupestre de Bolivia \\ (SIARB). La Paz, Bolivia. \\ Correo-e: plimatorrez@gmail.com
}

\author{
Claudia Rivera Casanovas \\ Universidad Mayor de San Andrés, Instituto de Investi- \\ gaciones Antropológicas y Arqueológicas (IIAA-UMSA) \\ - SIARB, La Paz, Bolivia. \\ ORCID: 0000-0003-3886-0340 \\ Correo-e: csrivera@umsa.bo \\ Freddy Taboada \\ Sociedad de Investigación del Arte Rupestre de Bolivia \\ (SIARB). La Paz, Bolivia. \\ Correo-e: taboadatellez@yahoo.com
}

Recibido: 17/3/2021 - Aprobado: 17/6/2021

Cómo citar: Strecker, M., Lima, P., Rivera Casanovas, C., \& Taboada, F. (2021). Registro, documentación e interpretación del arte rupestre. Una propuesta básica para estudiantes: el Seminario Taller de la SIARB. Ciencia y Sociedad, 46(3), 99-110. Doi: https://doi. org/10.22206/cys.2021.v46i3.pp99-110

\section{Resumen}

Los estudios de arte rupestre juegan un rol cada vez mayor dentro de los proyectos de investigación arqueológica. Se nota un incremento en los registros nacionales de sitios de pinturas y grabados rupestres, una siempre mayor aplicación de nuevas técnicas en su documentación, análisis empleando métodos de arqueometría, numerosos encuentros de especialistas en la temática, así como publicaciones especializadas. A pesar de todos estos avances, se advierte una falta de programas especializados en arte rupestre en el currículum académico de distintas universidades con programas de enseñanza e investigación relacionados con esta temática. En este artículo presentamos y explicamos el programa del Seminario Taller Introducción al Estudio del Arte Rupestre, organizado por la Sociedad de Investigación del Arte Rupestre de Bolivia (SIARB), dirigido a estudiantes de Arqueología, Antropología y otras

\begin{abstract}
Rock art studies play an increasingly important role in archaeological projects, as evidenced by national registers of rock engravings and paintings, the application of new technology in recording processes, analysis of the art by archaeometry, numerous academic events on rock art and specialized publications. However, rock art does not play an equal role in academic programs of universities in South America; bachelor theses on rock art are scarce compared to those on other archaeological and anthropological research topics. The authors of this article present and explain the program of the seminar workshop with introduction to rock art studies, organized by the Bolivian rock art research society SIARB, directed at students of archaeology, anthropology, history, arts, and tourism. It stresses recording of sites with simple methods that may be used by novices in rock art research, as well as
\end{abstract}


carreras (Artes, Historia, Turismo). Este programa hace énfasis en el registro y la documentación de sitios de arte rupestre con simples métodos adecuados para principiantes en la materia; asimismo, se incide en la conservación del arte rupestre, la clasificación e interpretación de los sitios y sus manifestaciones rupestres.

Palabras clave: arte rupestre, formación académica, registro, documentación, interpretación.

\section{Introducción}

A nivel mundial, los estudios de arte rupestre, que en el pasado fueron apenas tomados en cuenta dentro de proyectos arqueológicos, tienen un rol importante en el desarrollo de las investigaciones. Se observa un aumento en los registros nacionales de sitios de pinturas y grabados rupestres, una siempre mayor aplicación de nuevas técnicas en su documentación, análisis empleando métodos de arqueometría, numerosos encuentros de especialistas en la temática, además de publicaciones especializadas (Bahn, Franklin, Strecker \& Devlet, eds., 2016; Bahn, Franklin \& Strecker, 2021). A pesar de esto, en general, se advierte una falta de programas especializados en arte rupestre en el currículum académico de las universidades de Sudamérica.

Parte del problema en Latinoamérica es que son pocas las instituciones nacionales que, por un lado, se ocupen del registro y del estudio de sitios de arte rupestre de parte de equipos profesionales y, por otro lado, fomenten la capacitación de nuevos estudiantes e investigadores en este campo. Este tipo de instituciones existen, por ejemplo, en Sudáfrica (Rock Art Research Institute, Witwatersrand University) y Australia (Centre for Rock Art Research + Management - The University of Western Australia; Rock Art Heritage Unity, Griffith University; Australian National University). En este contexto, podemos destacar el rol asumido en Sudamérica por la Sociedad de Investigación del Arte Rupestre de Bolivia (SIARB), el Grupo de Investigación del conservation, classification and interpretation of the sites and the rock art.

Keywords: rock art, academic training, registration, recording, interpretation.

Patrimonio Rupestre (GIPRI), en Colombia, y el Instituto Nacional de Antropología y Pensamiento Latinoamericano (INAPL), en Argentina.

En las instituciones académicas sudamericanas que se ocupan de la enseńanza de antropología y arqueología, la Universidad de Buenos Aires (UBA, Depto. de Ciencias Antropológicas, Facultad de Filosofía y Letras) es una de las pocas que ha enfocado los estudios de arte rupestre, como nos hacen conocer las colegas Dánae Fiore, Anahí Re y María Pia Falchi (com. pers., diciembre de 2015). Entre los años 1980 y 2012 se realizaron nueve tesis de licenciatura sobre sitios de arte rupestre (Kligmann \& Ramundo, 2014, p. 258, Tabla 2); las primeras tres surgieron en la década de los años 90 y hubo un aumento considerable en los años siguientes, a partir del año 2000 (Kligmann \& Ramundo, 2014, p. 261, Tabla 5). Sin embargo, de 241 tesis analizadas, las nueve tesis que tratan la temática de arte rupestre ocupan un rango inferior de solo un 3,73\% (Kligmann \& Ramundo, 2014, p. 264, Tabla 6). Durante el año 2015, las catedráticas Anahí Re y María Pia Falchi dictaron un seminario de grado optativo para estudiantes de la carrera llamado "Representaciones rupestres: teoría, metodología y casos". Fueron 16 clases de 4 horas cada una, dictadas a lo largo de un cuatrimestre (A. Re, com. pers., diciembre de 2015). Hasta el presente, la UBA sigue sin tener programas especializados en arte rupestre. Cabe mencionar que se tiene en marcha un cambio de plan de estudios de la carrera de Ciencias Antropológicas (está en las últimas etapas de aprobación). En ese plan se incluye una materia optativa de 


\section{Registro, documentación e interpretación del arte rupestre. Una propuesta básica para estudiantes: el Seminario Taller de la SIARB}

"Arqueología del arte". Más allá de eso, actualmente la incorporación de los trabajos que abordan el arte rupestre en el currículo depende de las iniciativas de los docentes que trabajan en la UBA (A. Ré, com. pers., febrero de 2021). Por otro lado, los centros de investigación con sus programas de entrenamiento laboral tienen mucha importancia para la formación de los estudiantes; estos programas funcionan para todas las especialidades de arqueología, entre ellas el arte rupestre.

En Bolivia, el programa de estudios de las carreras de Arqueología y Antropología de la Universidad Mayor de San Andrés (UMSA) de la ciudad de La $\mathrm{Paz}$ no incluye el arte rupestre como parte de su oferta académica. Para los estudiantes es más difícil capacitarse en esta temática y son muy contadas las tesis de licenciatura que se refieren a sitios con pinturas o grabados rupestres como temas centrales de investigación. Entre 1992 y 2020 se produjeron 79 tesis de licenciatura, de ellas, solo cuatro tratan de manera significativa temas referidos al arte rupestre $(5,06 \%)$, mostrando la llamativa ausencia que existe en la investigación sobre la temática rupestre.

Dentro de este contexto de formación académica arqueológica y de la arqueología misma en Bolivia, las iniciativas educativas de la Sociedad de Investigación del Arte Rupestre de Bolivia (SIARB), en particular nuestro seminario taller, que ofrece una introducción al estudio del arte rupestre para estudiantes universitarios, llena un vacío dentro de estos programas de enseñanza universitaria.

A nivel de Sudamérica, conocemos solamente una institución más que ofrece regularmente cursillos sobre arte rupestre: GIPRI, en Colombia. En su quinto seminario taller participó la experta norteamericana Jane Kolber, quien enseńó métodos básicos de registro y documentación (Kolber, 2017). Durante dos días del mes de julio de 2019, GIPRI realizó su VII Seminario Taller de Arte Rupestre enfocando las temáticas de estudios interdisciplinarios y diagnóstico de conservación.
Considerando este contexto a nivel sudamericano es que se presenta la experiencia de la SIARB como institución gestora en la formación de estudiantes interesados en temáticas de arte rupestre.

\section{Marco teórico}

Los conceptos del patrimonio cultural y de la gestión patrimonial son dinámicos, dependen de la sociedad actual y de los valores asignados a los componentes del patrimonio cultural, por ende, están en constante transformación; respecto a estos conceptos, Jiménez-Esquinas (2020) comenta: "han ido variando en las últimas décadas hacia un abordaje antropológico de cultura y un mayor protagonismo de las comunidades" (p. 100); agrega: "hasta un progresivo acercamiento al concepto antropológico de cultura centrado en la diversidad, en lo holístico y procesual y con un mayor protagonismo de los sujetos", de manera que la participación social de las comunidades, los grupos y, en algunos casos los individuos, se ha erigido como un requisito imprescindible para elevar cualquier candidatura a la lista de patrimonio inmaterial de la UNESCO (Jiménez-Esquinas, 2020, p. 103). Entre los distintos modelos de relación arqueología-sociedad está la "arqueología pública”, desde los años 1970, cuyo objetivo, según el referido autor, es "que la sociedad se involucre de tal forma que llegue a contemplar la destrucción de este recurso finito como una agresión a su derecho a la cultura y una pérdida para los Estados en su conjunto" (p. 104). Sin embargo, en este modelo no se ha considerado una participación activa de las comunidades, que sí fue la base de modelos posteriores que surgieron a partir de los ańos ochenta del siglo pasado, favoreciendo, finaliza el autor, "la incorporación de voces, prácticas y saberes como los de las mujeres, grupos indígenas, grupos minoritarios, marginalizados y también actores no-académicos, cuyas interpretaciones del pasado habían sido históricamente relegadas del discurso arqueológico, lo cual recibió el nombre de multivocalidad" (p. 107). En este sentido, Brian Egloff (2019) enfatiza en su visión de los procesos y factores de conservación y administración de sitios arqueológicos los 
derechos indígenas respecto a los sitios patrimoniales y la participación de comunidades; por ejemplo, se refiere al Código de Ética de la Asociación Arqueológica de Australia que exige que en cualquier proyecto los interesados (stakeholders) de las comunidades aborígenes deben dar su aprobación de los trabajos y deben recibir los informes que resulten del proyecto. Estos enfoques juegan un papel importante en los proyectos desarrollados por la Sociedad de Investigación del Arte Rupestre de Bolivia (SIARB), que considera a los actores locales (comunidades y alcaldías o gobiernos municipales) como los principales responsables de la preservación y administración de los sitios. En tal sentido, se considera que deben participar activamente en cada una de las fases del proyecto, así como en la toma de decisiones.

Respecto al registro arqueológico, compartimos la noción de Basso (2021, p. 108) que "conforma parte de un paisaje que puede ser abordado a partir de una estrategia de investigación que comprende el estudio de todos los procesos sociales e históricos..., tornándose en el marco adecuado para la investigación de la vida social a largo plazo". Además, Basso (2021) argumenta que -tomando en cuenta que existen representaciones rupestres de contextos de distintos períodos cronológicos, tanto de los períodos prehispánicos, como de la Colonia y la República- "para poder responder a un interrogante mayor acerca de procesos de continuidades y/o cambios en algunas prácticas de la etapa prehispánica en relación a la colonial, el principal abordaje teórico se realiza desde conceptos de la Arqueología Histórica teniendo en cuenta que el período Colonial es parte de procesos mayores de la historia de una localidad".

Por otro lado, nuestra visión del arte rupestre encaja en la llamada Arqueología del Paisaje, entiendo "paisaje" como concepto fluido que incluye una perspectiva dinámica en la que múltiples procesos y sistemas interactúan (Reher Díez, 2019, pp. 184-194), ya que consideramos las manifestaciones rupestres en sus lugares naturales como parte de los paisajes culturales de los pobladores antiguos y actuales. En este resumen, el paisaje, "aunque se sustenta en un soporte material, se define ante todo como una red o trama, un complejo sistema de relaciones subjetivas y ecosistémicas: sociales, productivas, medioambientales y también históricas y culturales" (Burgos Barrantes, 2020, p. 34).

Mientras las observaciones anteriores se refieren al marco teórico de la investigación de diversas clases de sitios arqueológicos, deseamos destacar algunos conceptos teóricos específicos respecto al estudio del arte rupestre, que -según Bruno David e Ian McNiven (2018) - ganaron mucho más importancia a partir de la década de 1980, debido a la posibilidad de aplicar nuevos métodos de datación AMS de muestras de tamaño diminuto. A la vez, nuevos marcos teóricos se desarrollaron desde el llamado procesionalismo (la idea de que los arqueólogos deberían estudiar en primer lugar los procesos de creación de restos culturales para entender el comportamiento social de cada cultura) hacia los estudios de comportamiento simbólico y las maneras muy diversas en que los pueblos entienden el mundo. Por otro lado, según Jo McDonald \& Peter Veth (2012), a partir de los primeros años de este milenio surgieron nuevos enfoques en la investigación del arte rupestre, tales como: la llamada "fenomenología", que explora las maneras en que el arte rupestre fue concebido, producido, colocado y modificado a través del tiempo; la hermenéutica, que busca aclarar el significado de las representaciones rupestres considerando su contexto social; además, la documentación y el análisis con nuevas tecnologías digitales.

Finalmente, respecto a la conservación de sitios de arte rupestre, Neville Agnew y sus colegas (2015) postularon los siguientes ocho principios: la enérgica promoción de la conservación, reclamando los recursos para proyectos profesionales (en la realidad 


\section{Registro, documentación e interpretación del arte rupestre. Una propuesta básica para estudiantes: el Seminario Taller de la SIARB}

latinoamericana prácticamente imposible); la consideración de todos los valores culturales; la preservación y administración del arte rupestre como parte del paisaje; el respeto y el mantenimiento de derechos y prácticas culturales (incluye, por ejemplo, ritos ancestrales realizados en los sitios, como en el caso de una comunidad en el departamento de Oruro, Bolivia); el empoderamiento de los propietarios indígenas tradicionales y las comunidades locales y su inclusión en las decisiones respecto a la administración y conservación de los sitios; el uso de métodos de documentación, conservación e interpretación de acuerdo a protocolos y normas internacionales; prioridad a la conservación preventiva (que incluye campañas educativas); comunicación efectiva y colaboración con la comunidad local como clave para el éxito de la administración del sitio. Este último punto es fundamental en trabajos recientes en la conservación y administración de sitios de arte rupestre, como destacan M. Strecker \& F. Taboada (2021) en su revisión de cinco proyectos en Argentina, Bolivia y México.

Tomamos en cuenta estas corrientes actuales en nuestra capacitación de estudiantes de arqueología y antropología, aunque el énfasis en nuestras actividades educativas es, en primer lugar, una valoración del arte rupestre como parte del patrimonio cultural y una sensibilización sobre la necesidad de preservar estos sitios.

\section{Educación sobre el patrimonio cultural de arte rupestre}

El modelo actual de una "arqueología comunitaria", a la que nos hemos referido, tiene consecuencias respecto a la enseńanza sobre los sitios de arte rupestre, vista desde la perspectiva de los actores locales. En este sentido, existen publicaciones notables en Perú (Hostnig, 2009) y en Argentina (Falchi, 2014) que presentan el arte rupestre regional a la población local, en particular a alumnos y estudiantes de cole- gios. Asimismo, las campañas educativas de la SIARB (ver abajo) tratan de comunicar la importancia del arte rupestre como parte del patrimonio cultural a la población en general, a niños, estudiantes, así como a miembros de comunidades y gobiernos locales, los cuales son responsables de la gestión de parques arqueológicos con arte rupestre.

El contexto antes descrito también permite constatar deficiencias notables en los programas educativos sobre el patrimonio cultural nacional en la mayoría de los países latinoamericanos, en particular con respecto al arte rupestre. En el caso de Argentina, Fiore et al. (2016) presentan un análisis sistemático de libros de escuelas usados entre 1910 y 2010, respecto a sus contenidos sobre arte rupestre. Este análisis permitió identificar que gran parte de los libros sobre arte rupestre está relacionado con el paleolítico europeo, desconociendo los sitios de pinturas y grabados rupestres en el territorio nacional.

En ese entendido, y en el marco de la participación activa de los actores locales, la SIARB implementa también programas de sensibilización y capacitación en las comunidades y/o poblaciones en las que implementa sus proyectos. El objetivo central de estos programas es promover la preservación y conservación del patrimonio cultural local, arte rupestre en este caso, a través del conocimiento y de la educación. En vista de que los programas educativos oficiales no consideran estos aspectos, y a pesar de que la gente conoce los sitios de arte rupestre, se advierte poca empatía con el valor patrimonial y la preservación de estos sitios. Por tanto, los talleres impartidos están enfocados en metodologías participativas, las que promueven y fomentan la apropiación de los temas que se presentan, logrando que la gente contribuya con su conocimiento y sus experiencias (Carrasco, 2017). De esta manera, se logra un proceso de retroalimentación del conocimiento técnico y local. 


\section{Campańas educativas de la SIARB}

A lo largo de más de 30 años de trabajo, la SIARB ha desarrollado diferentes campañas educativas dirigidas a:

- los mismos investigadores del arte rupestre que -según nuestro Código de Ética-deben respetar la integridad de los sitios, trabajar en proyectos legalmente establecidos y evitar métodos de documentación que podrían afectar la conservación del arte rupestre,

- estudiantes que deseen recibir una introducción al estudio del arte rupestre y que participan en eventos como el cursillo sobre registro y documentación del arte rupestre (Tarija, diciembre de 2000) y nuestro seminario taller (ver abajo),

- profesores de escuelas y colegios, nińos, jóvenes y público en general, por ejemplo a través de nuestra hoja didáctica “¿Qué es el arte rupestre?", una serie de exposiciones en museos y otras instituciones culturales; finalmente, a través de nuestra página web www.siarb-bolivia.org, que incluye una amplia sección tratando temas de educación sobre arte rupestre,

- miembros de comunidades y gobiernos municipales, guías y guarda ruinas de parques arqueológicos con arte rupestre, a través de talleres,

- visitantes de sitios de arte rupestre, mediante folletos y un video.

De esta manera, se hizo visible el trabajo de esta institución en el afán de socializar la importancia de la protección y conservación del arte rupestre en el contexto de las poblaciones locales y de las entidades de gestión patrimonial del Estado. Un paso posterior fue la realización de cursos especializados, dirigidos a profesionales y estudiantes, en los cuales se analizaron técnicas de registro, procesos de gestión del arte rupestre y su relación e impor- tancia interdisciplinaria. Todos estos aspectos son descritos a continuación.

\section{El Seminario Taller Introducción al Estudio del Arte Rupestre}

En julio de 2010, la SIARB ofreció por primera vez un seminario taller sobre los estudios de arte rupestre para estudiantes de Arqueología, Antropología y otras carreras (Artes, Historia, Turismo); este evento se realizó con el coauspicio de varias instituciones y contó con la participación de estudiantes de Bolivia y Perú. En julio de 2013 se realizó en La Paz un segundo seminario taller parecido, con estudiantes internacionales. En abril de 2016 organizamos este evento por tercera vez en instalaciones del Instituto de Investigaciones Antropológicas-Arqueológicas (IIAA), UMSA, unidad académica que hizo posible este curso dentro de un contexto académico universitario; en abril de 2018 se realizó la cuarta versión del seminario taller en la misma casa de estudios en coordinación con el IIAA. Para cada versión de este evento los contenidos fueron modificados y actualizados.

Considerando que la temática de arte rupestre no forma parte del currículum ofrecido en las Carreras de Antropología y Arqueología de la UMSA ni del de otras carreras, como Historia, Artes Plásticas o Turismo, la Sociedad de Investigación del Arte Rupestre de Bolivia planifica nuevamente la realización de este curso durante el año 2021.

La metodología diseñada consiste en la implementación de actividades teóricas y prácticas en forma interactiva, con participación activa de los estudiantes. Se imparten clases en aula, complementadas por un trabajo de campo, para lo cual se realiza una excursión donde se ponen en práctica parte de los contenidos teóricos impartidos. Gran parte de los contenidos es entregado también a los estudiantes, quienes reciben diversos materiales como fotocopias. De igual manera, se entregan certificados a los participantes que hayan 


\section{Registro, documentación e interpretación del arte rupestre. Una propuesta básica para estudiantes: el Seminario Taller de la SIARB}

asistido puntualmente a todas las clases y actividades del seminario.

El curso fue diseñado considerando la pedagogía moderna, enfocada en el planteamiento de objetivos claros y en el desarrollo de competencias en los procesos educativos. En ese sentido, los objetivos principales están centrados en transmitir conocimientos teóricos, metodológicos y prácticos sobre el arte rupestre para generar varias competencias educativas entre los estudiantes, como puede verse con detalle en la Tabla 1.

\section{Tabla 1}

Objetivos y competencias del Seminario Taller Introducción al Arte Rupestre

\begin{tabular}{ll}
\hline Objetivos & Competencias \\
\hline & - Los estudiantes sabrán definir el arte rupestre por sus características materiales (soporte y técnicas). \\
& - Entenderán la importancia del arte rupestre como fenómeno cultural, parte del patrimonio cultural. \\
& - Entenderán que la investigación del arte rupestre incluye diferentes especialidades (geología, \\
& arqueología, etnografía, conservación, etc.). \\
Objetivo 1: Impartir & - Conocerán ejemplos de arte rupestre paleolítico europeo. \\
conocimientos teóricos sobre & - Podrán explicar diferentes posibilidades de datación aproximada del arte rupestre. \\
arte rupestre y su estudio en & - Conocerán ejemplos de interpretación etnográfica del arte rupestre. \\
general & - Aprenderán métodos de análisis, descripción e interpretación tentativa del corpus de arte rupestre de \\
& - En sitio o un conjunto de sitios. \\
& - Podrán reconocer y explicar el impacto del turismo "salvaje" (sin control y administración) a sitios \\
& arqueológicos y de arte rupestre.
\end{tabular}

Objetivo 2: Generar conocimientos teóricos sobre arte rupestre de Sudamérica y de Bolivia en particular
- Los estudiantes conocerán ejemplos del arte rupestre más antiguo en Sudamérica.

- Conocerán ejemplos del arte rupestre de Bolivia.

- Podrán distinguir entre varias tradiciones en el arte rupestre de Bolivia tomando en cuenta sus características formales: soporte, técnica de ejecución, tamaño, características estilísticas, motivos representados.
Objetivo 3: Dar a conocer información teórica sobre turismo y parques arqueológicos

Objetivo 4: Lograr conocimientos prácticos para el registro de sitios de arte rupestre
- Los estudiantes conocerán algunos ejemplos de parques arqueológicos en Europa, EE. UU. y Bolivia.

- Manejarán un modelo para proyectos de puesta en valor de sitios de arte rupestre.
Objetivo 5: Analizar criterios para la realización de trabajos académicos sobre arte rupestre, en particular tesis de Licenciatura en Arqueología y Antropología

Objetivo 6: Reflexionar sobre los contextos culturales y significados del arte rupestre a partir de distintas aproximaciones
- Los estudiantes podrán aplicar varias formas de registro que no dañen al arte rupestre o su soporte.

- Se darán cuenta de la imposibilidad de registrar un sitio y sus elementos fielmente a través de un solo método de documentación.
- Los estudiantes reflexionarán sobre enfoques específicos de tesis sobre arte rupestre que toman en cuenta procesos socioeconómicos.

- Analizarán tesis sobre arte rupestre, constatando fortalezas, debilidades y aportes. 
Como complemento, en sus visitas a sitios con arte rupestre, los estudiantes analizan los objetivos y métodos empleados, así como se observan in situ los resultados de proyectos de investigación.

El programa consta de cuatro días de clases en aula más un día de trabajo de campo en sitios de arte rupestre. Adicionalmente, se tiene acceso a estudios individuales con materiales, sumando un total aproximado de 40 horas de trabajo.

Trabajamos con un equipo de docentes que incluyen arqueólogos, especialistas en arte rupestre, documentación y conservación de sitios. Aparte de miembros de la SIARB, Prof. MatthiasStrecker, Dra. Claudia Rivera, Lic. Pilar Lima, Lic. Freddy Taboada y Lic. Genaro Huarita, incluimos aportes en forma de fotos, materiales e inclusive presentaciones completas de parte de: Dr. Pablo Cruz (Argentina), Dr. Lawrence Loendorf (EE. UU.), Dra. Tamara Bray (EE. UU.), Dr. Robert Mark (EE. UU.), Jane Kolber (EE. UU.), Jon Harman (EE. UU.), Jeff LaFave (EE. UU.), Dra. Elizabeth Arkush (EE. UU.), Dr. Paul Bahn (Gran Bretaña), J. I. Royo Guillén (España), Dr. Michel Lorblanchet (Francia), Dr. Tilman Lenssen-Erz (Alemania), David Welch (Australia), Rainer Hostnig (Perú), Dr. André Prous (Brasil), Dra. M. F. Ugalde (Ecuador), Lilo Methfessel (Tarija), Carlos Kaifler (Santa Cruz), Lic. Rosario Saavedra (Potosí), GIPRI (Colombia) y otros.

A continuación, especificamos brevemente los contenidos de los módulos impartidos en el seminario taller (Tabla 2).

\section{Tabla 2}

\section{Contenidos de los módulos impartidos en el Seminario Taller Introducción al Arte Rupestre}

\begin{tabular}{|c|c|}
\hline Módulo & Contenido \\
\hline $\begin{array}{l}\text { Módulo 1: } \\
\text { Aspectos } \\
\text { conceptuales, } \\
\text { marco general del } \\
\text { arte rupestre y } \\
\text { conservación }\end{array}$ & $\begin{array}{l}\text { - Arte rupestre: definición, técnicas, sitios. } \\
\text { - El estudio del arte rupestre como investigación multidisciplinaria (geología, geografía, arqueología, } \\
\text { conservación, etc.). } \\
\text { - Analizamos el arte rupestre: categorías analíticas y descriptivas (soporte, técnicas de ejecución, motivos, } \\
\text { elementos). } \\
\text { - La conservación del arte rupestre y el impacto del turismo sin control. }\end{array}$ \\
\hline $\begin{array}{l}\text { Módulo 2: } \\
\text { Arte rupestre e } \\
\text { historia-arqueología }\end{array}$ & $\begin{array}{l}\text { - El rol del arte rupestre en la historia del arte (arte rupestre paleolítico). } \\
\text { - Arte rupestre y arqueología - problemas de cronología / datación / el arte rupestre como parte integral del } \\
\text { pasado de las sociedades / excavaciones en Intinqala, Copacabana. } \\
\text { - El arte rupestre más antiguo en Sudamérica. }\end{array}$ \\
\hline $\begin{array}{l}\text { Módulo 3: } \\
\text { Arte rupestre en } \\
\text { Bolivia }\end{array}$ & - Ejemplos de tradiciones del arte rupestre en Bolivia. \\
\hline $\begin{array}{l}\text { Módulo 4: } \\
\text { Etnografía y arte } \\
\text { rupestre }\end{array}$ & $\begin{array}{l}\text { - Consideraciones generales. } \\
\text { - Ejemplos del SO de Norteamérica. } \\
\text { - Ejemplos de Bolivia. } \\
\text { - La construcción del paisaje en regiones campesinas del área surandina. }\end{array}$ \\
\hline $\begin{array}{l}\text { Módulo 5: } \\
\text { Turismo y arte } \\
\text { rupestre }\end{array}$ & $\begin{array}{l}\text { - Consideraciones generales - Ejemplos de parques arqueológicos con arte rupestre en Europa y EE. UU. } \\
\text { - Ejemplos de parques arqueológicos con arte rupestre en Bolivia. }\end{array}$ \\
\hline $\begin{array}{l}\text { Módulo 6: } \\
\text { Registro y } \\
\text { documentación del } \\
\text { arte rupestre }\end{array}$ & $\begin{array}{l}\text { - Registro y documentación del arte rupestre (métodos / fichas / etc.). } \\
\text { - Aplicación del programa DStretch a fotografías de arte rupestre. }\end{array}$ \\
\hline $\begin{array}{l}\text { Módulo 7: } \\
\text { Tesis sobre arte } \\
\text { rupestre }\end{array}$ & $\begin{array}{l}\text { - Presentación y análisis de varias tesis de Licenciatura sobre arte rupestre. } \\
\text { - Recursos para los estudios del arte rupestre (bibliografía básica / internet / archivo y biblioteca de la SIARB). }\end{array}$ \\
\hline
\end{tabular}


Además, se explican las bases teóricas del trabajo de campo y se explican las tareas que se deben cumplir en un sitio de arte rupestre. Finalmente, se realiza un trabajo de campo y una excursión a sitios de pinturas rupestres en la Prov. Los Andes, Depto. La Paz. Se eligió esta área para el taller, debido a la cantidad de sitios importantes de arte rupestre y a la cercanía de los sitios con relación a la ciudad de La Paz.

\section{Figura 1}

Participantes del seminario taller del año 2016 en un sitio de arte rupestre

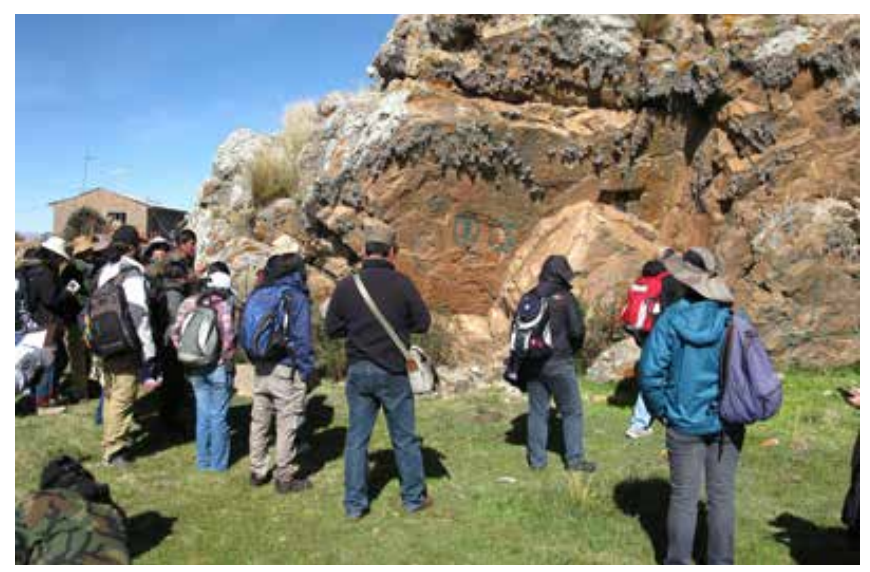

Nota. Foto: Archivo SIARB.

\section{Figura 2}

Participantes del seminario taller del año 2018 en un sitio de arte rupestre

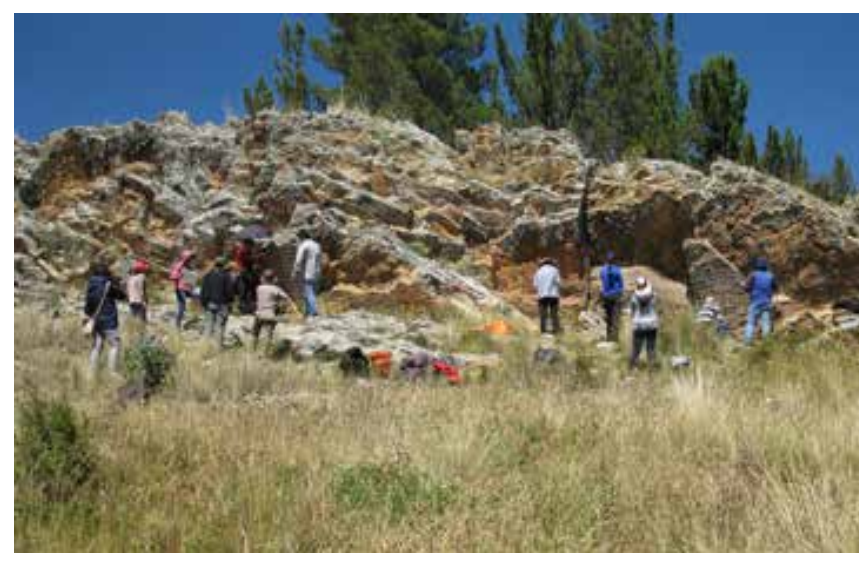

Nota. Foto: Archivo SIARB.

\section{Figura 3}

Participantes del seminario taller del año 2016 en un sitio de arte rupestre

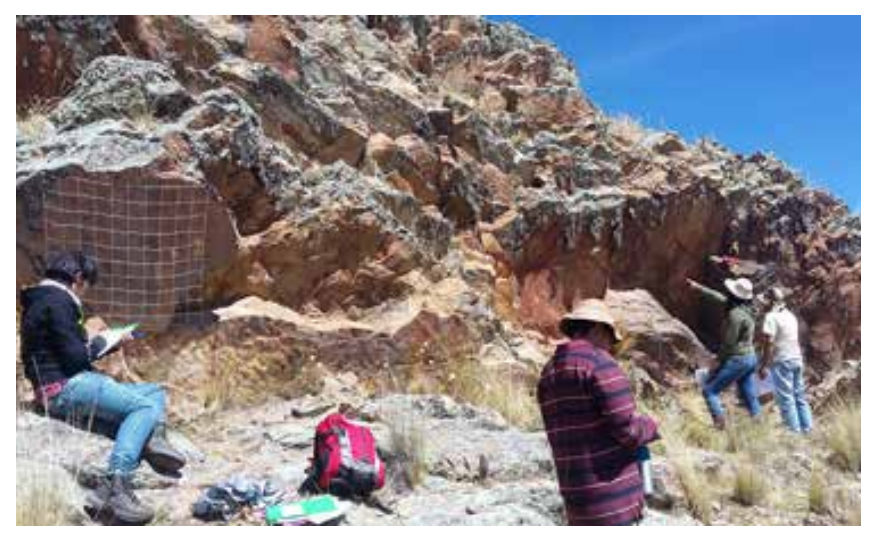

Nota. A la izquierda red que facilita el dibujo a escala. Foto: Archivo SIARB.

En este programa existe cierto énfasis en el registro y la documentación de sitios de arte rupestre con simples métodos, adecuados para principiantes en la materia. Este hecho refleja nuestra convicción de que la base de cualquier análisis o interpretación de un sitio de arte rupestre debe ser una documentación confiable.

De igual manera, se incide en la conservación del arte rupestre, se orienta que los estudiantes tomen conciencia de la fragilidad de este patrimonio cultural y que se entienda que el arte rupestre es un sistema complejo y dinámico, cuya estabilidad depende de las relaciones de tres aspectos fundamentales: a) los factores intrínsecos del soporte y del pigmento, b) los factores naturales del medio ambiente, y c) la acción antrópica.

A finalizar el evento, se hace una evaluación con los estudiantes sobre cuáles contenidos fueron particularmente importantes para ellos. En los talleres ya implementados, los estudiantes -en general- se mostraron muy contentos con lo aprendido, expresando que desearían recibir prácticas más intensivas en el registro y la documentación en sitios de grabados y pinturas rupestres. 
Lamentablemente, esta demanda no puede ser atendida como los estudiantes esperan debido al tiempo disponible, que debe ser coordinado con la Carrera de Antropología y Arqueología de la UMSA. A esto se suma la reducida cantidad de recursos de la SIARB en actuales proyectos, aspecto que no permite aprovechar estas actividades sistemáticamente para la capacitación de estudiantes. Sin embargo, sabemos que varios estudiantes aprovecharon los conocimientos básicos adquiridos en nuestro seminario taller para continuar el trabajo y especializarse en esta temática.

Como se mencionó anteriormente, todo el proceso implementado en las diferentes versiones del seminario taller con estudiantes universitarios fue también replicado a nivel de las poblaciones locales, aunque a menor escala. Todos los proyectos que la SIARB implementó para la conformación de parques arqueológicos o de conservación de sitios de arte rupestre tuvieron siempre un componente de sensibilización y capacitación de actores locales. En estos talleres se trató de lograr sensibilidad en los pobladores de un área para promover la preservación de los sitios de arte rupestre, intentando evitar el vandalismo y promoviendo una valoración cultural para el patrimonio local. De esta forma, el tratamiento de temas vinculados al arte rupestre es transversal, tanto a un grupo especializado -como son los estudiantes- como a los detentores de este patrimonio.

Las diferentes versiones de cursos implementadas por la SIARB, así como los talleres impartidos en más de 30 años de trabajo, se constituyen en un aporte significativo en Bolivia para el tratamiento de esta temática.

En los EE.UU. existe un modelo diferente en forma de "escuelas de campo" (field schools) que logran a la vez la capacitación de documentalistas y el registro y la documentación de numerosos sitios de arte rupestre. Entre 1975 y 1980, la sociedad arqueológica de New Mexico organizó cada verano su "Rock Art Field School" y documentó una parte conside- rable del arte rupestre de Chaco Canyon; entre 1987 y 1992, se continuó de la misma manera con la documentación completa del extenso sitio de petroglifos Three Rivers ${ }^{1}$. Otras instituciones norteamericanas, nos referimos a varias universidades, siguieron estos ejemplos. La Universidad de Arizona (School of Anthropology) ha organizado su programa "Rock Art Ranch Field School" desde 2011. También se organizan actividades parecidas con estudiantes y/o voluntarios en Gran Bretaña -en Inglaterra y Escocia $^{2}$, en Valcamonica (Italia) y en Australia (Flinders University y Australian National University); en este último caso se trata de una colaboración entre académicos, voluntarios y comunidades aborígenes. Obviamente, las llamadas escuelas de campo son muy atractivas y pueden ofrecer una capacitación intensiva, si existen las condiciones adecuadas de parte de instituciones académicas y de comunidades. En el futuro habrá que hacer esfuerzos para organizarlas también en Bolivia y otros países sudamericanos.

\section{Conclusiones}

Considerando las condiciones descritas previamente, la experiencia desarrollada por la SIARB permite identificar algunos resultados y algunas conclusiones que fueron producto de los talleres impartidos:

- El haber participado de los seminarios taller permitió que algunos estudiantes, tanto en Bolivia como en el exterior, realizaran sus tesis de licenciatura en temáticas relacionadas al arte rupestre.

- La realización de estos talleres permite la actualización de los contenidos temáticos, trabajo que también implica la actualización de las personas que imparten el taller.

1. Fuente: https://humansystemsresearch.org/publications/threerivers-petroglyph-site-results-of-the-rock-art-recording-fieldschool/

2. Ver: https://www.rockart.scot/about-us/blog/volunteeringwith-scrap-from-kilmartin-field-school-and-beyond/ 


\section{Registro, documentación e interpretación del arte rupestre. Una propuesta básica para estudiantes:}

el Seminario Taller de la SIARB

- Los talleres, impartidos en el ámbito universitario bajo un acuerdo de cooperación con el Instituto de Investigaciones Antropológicas y Arqueológicas (IIAA) de la Universidad Mayor de San Andrés, constituyen una experiencia innovadora dentro del ámbito académico universitario, complementando la formación de los estudiantes y abriendo un espacio para la participación de profesores universitarios dentro de la temática del arte rupestre en Bolivia.

- El tener una perspectiva crítica de los estudiantes con relación a los trabajos que realiza la SIARB en Bolivia permite considerar los aspectos mencionados en futuros trabajos. De esta forma, las actividades del seminario taller se convierten también en un proceso de retroalimentación que es saludable a nivel institucional.

- Todo el proceso implementado por la SIARB para dar a conocer el arte rupestre y promover su preservación, en más de 30 años, se convierte en un gran esfuerzo de gestión del patrimonio cultural de Bolivia.

- Finalmente, la realización de eventos virtuales de difusión general sobre temas en el arte rupestre de Bolivia, considerando las oportunidades y limitaciones en tiempos de pandemia (2020) y a futuro, abren nuevas oportunidades de incorporar las experiencias de la SIARB en un contexto de enseñanza-aprendizaje mediante el uso de algunas herramientas virtuales de educación a distancia.

\section{Referencias}

Bahn, P., Franklin, N., \& Strecker, M. (Eds.) (2021). Rock Art Studies: News of the World. Vol. 6. Archaeopress Archaeology (en prep.).

Bahn, P., Franklin, N., Strecker, M., \& Devlet, E. (Eds.) (2016). Rock Art Studies: News of the World. Vol. 5. Archaeopress Archaeology.

Basso, D. M. (2021). Estudios sobre cambios y continuidades entre el periodo Prehispánico y la etapa Colonial en la localidad de Cochinoca (Puna de Jujuy). A la memoria de María Ester Albeck. Mundo de Antes, 15(2), 103-134. Instituto de Arqueología, Universidad de Tucumán. Burgos Barrantes, B. (2020). De los objetos a los sujetos: transiciones del patrimonio cultural. Reconceptualizaciones y reinstitucionalizaciones. Revista PH101, Especial Monográfico, De lo público al bien común: emergencia de otros modelos de gestión del patrimonio cultural, 26-47.

Carrasco, P. (2017). Guía de metodologias participativas para facilitadores de grupos. Departamento de participación Social y Gestión de usuario, SSMSO.

David, B., \& McNiven, I. (2018). Introduction. Towards an archaeology and anthropology of rock art. The Oxford Handbook of the Archaeology and Anthropology of Rock Art (B. David e I. McNieven, eds.): 1-21. Oxford University Press.

Egloff, B. (2019). Archaeological Heritage Conservation and Management. Archaeopress.

Falchi, M. P. (2014). Los colores de la prehistoria. Guachipas. INAPL.

Fiore, D., Ocampo, M., \& Acevedo, A. (2016). Teaching and Learning about Rock Art in Argentina. Relating to Rock Art in the Contemporary World: symbols, meaning, significance (L. M. Brady y P. S. C. Taçon, eds.), 275-305. University Press of Colorado.

Hostnig, R. (2009). Los petroglifos de Boca Chaquimayo, San Gabán. Testimonio cultural de la Amazonia puneña. Intersur Concesiones S.A.

Jiménez-Esquinas, G. (2020). El papel de las comunidades en el patrimonio: una reflexión en torno al patrimonio arqueológico. Revista $\mathrm{PH}$, 101, 100-121.

Kligmann, D. M. \& Ramundo, P. S. (2014). ¿Qué nos cuentan las actas de defensa de las tesis de licenciatura en ciencias antropológicas (orientación arqueológica) de la Facultad de Filosofía y Letras de la Universidad de Buenos Aires? Relaciones de la Sociedad Argentina de Antropología, 39(1), 245-276. 
Kolber, J. (2017). Colombia’s fifth rock art workshop and seminar. La Pintura, 43(3), 7-8.

McDonald, J., \& P. Veth (2012). Research issues and new directions: one decade into the new millenium. A Companion to Rock Art (J. McDonald y P. Veth, eds.), 1-14. Wiley-Blackwell, Australian University.

Reher Díez, G.-S. (2019). Los paisajes culturales como transferencia del conocimiento. Una solución para los retos actuales de la arqueología. [Tesis de doctorado, Universidad Complutense de Madrid].
Strecker, M. \& Taboada, F. (2021). Conservación y Administración de Sitios de Arte Rupestre - nuevos enfoques. Comentarios iniciales. Conservación y Administración de Sitios de Arte Rupestre - nuevos enfoques. Dossier de la revista Arqueología, Universidad de Buenos Aires. 